\title{
Boundary value problems for modified Helmholtz equations and applications
}

Longfei Gu and Zunwei Fu*

"Correspondence: fuzunwei@lyu.edu.cn

Department of Mathematics, Linyi University, Linyi, Shandong 276005, P.R. China

\section{Springer}

\begin{abstract}
We investigate factorizations of modified Helmholtz equations in Clifford algebra $\mathrm{Cl}\left(V_{3,3}\right)$. Using the method of fundamental solutions for modified Helmholtz equations and Clifford calculus, we obtain some integral representation theorem in Clifford analysis. The boundedness of singular integral operators in Hölder space is given. Moreover, we establish solvability conditions of Riemann type problems for modified Helmholtz equations in Clifford analysis. As applications, we solve a kind of singular integral equations. The explicit representation of the solution is also given.
\end{abstract}

MSC: $30 \mathrm{G} 35$

Keywords: Clifford algebra; Riemann type problem; Hölder space; modified Helmholtz equations

\section{Introduction}

As we know, the Helmholtz equations can be regarded as a generalization of the SturmLiouville equation to higher dimensions. The research on the Helmholtz equations $\Delta u \pm$ $\kappa^{2} u=0$ has drawn the attention of some physicists and mathematicians, we refer to [117]. Using a new transform method, Fokas, ben-Avraham and Antipov translate some important boundary value problems for linear and for integral nonlinear partial differential equations in physical plane to the corresponding modified Helmholtz equations. Novel integral representations for the solution of the Helmholtz and the modified Helmholtz equations formulated in the interior of a convex polygon are presented. These representations provide the basis for the development of certain analytical and numerical techniques for diffusion-limited coalescence, see [4-6] for more details. This article focuses on modified Helmholtz equations in Clifford analysis.

The Clifford approach is a powerful mathematical tool for the treatment of partial differential equations in higher dimensions, see [1-3, 7, 15-23]. Maxwell's equations in physics are the fundamental equations of electromagnetism and are recast into Helmholtz equations by using the Clifford approach, which is different from the vector calculus method. The electric and magnetic fields are treated together, both encoded as bi-vector into one part of a four-dimensional Clifford number in Clifford approach; we mention here [7, 1214]. It is natural to consider boundary value problems theory for Helmholtz equations and modified Helmholtz equations in higher dimensions, for instance, Riemann type problems, Dirichlet type problems, and so on. Besides the pure mathematical interest, these results are necessary for concrete problems in physics and engineering [8, 9]. In [17], Rie-

(c) $2015 \mathrm{Gu}$ and Fu. This article is distributed under the terms of the Creative Commons Attribution 4.0 International License (http://creativecommons.org/licenses/by/4.0/), which permits unrestricted use, distribution, and reproduction in any medium, provided you give appropriate credit to the original author(s) and the source, provide a link to the Creative Commons license, and indicate if changes were made. 
mann type problems for Helmholtz equations in the framework of Clifford algebra $\operatorname{Cl}\left(V_{n, 0}\right)$ are considered. Based on some ideas from [17], Riemann type problems for Helmholtz equations in Hermitian Clifford analysis are studied in [3]. However, to the best of our knowledge, some boundary value problems for modified Helmholtz equations and their applications in integral equations in the framework of Clifford algebra $\operatorname{Cl}\left(V_{n, n}\right)(n \geq 3)$ have not been considered. The main motivation is that the modified Helmholtz operator has been exactly factorized by means of the so-called $\pm \kappa$-Dirac operators $(\kappa>0)$ i.e., $\Delta-\kappa^{2}=(D+\kappa)(D-\kappa)$ in the Clifford algebra $C l\left(V_{3,3}\right)$.

In this article, motivated by [24, 25], in the framework of Clifford algebra $\operatorname{Cl}\left(V_{3,3}\right)$, we obtain second order generalized integral representations and solve some Dirichlet type problems for modified Helmholtz equations. We define some integral operators which are the generalization of classical Cauchy type integral operators, Teodorescu operators in Clifford analysis, and we study some properties of them. Finally, we study Riemann type problems for modified Helmholtz equations and give some applications.

\section{Preliminaries}

Let $V_{3,3}$ be an 3-dimensional real linear space with basis $\left\{e_{1}, e_{2}, e_{3}\right\}, C l\left(V_{3,3}\right)$ be the Clifford algebra over $V_{3,3}$ and the 8-dimensional real linear space with basis

$$
\left\{e_{A}, A=\left\{l_{1}, \ldots, l_{r}\right\} \in \mathcal{P} N, 1 \leq l_{1}<\cdots<l_{r} \leq 3\right\}
$$

where $N$ stands for the set $\{1,2,3\}$ and $\mathcal{P} N$ denotes the family of all order-preserving subsets of $N$ in the above way. Now denote $e_{\emptyset}$ by $e_{0}$ and $e_{l_{1} \ldots l_{r}}$ by $e_{A}$ for $A=\left\{l_{1}, \ldots, l_{r}\right\} \in \mathcal{P} N$. The product on $C l\left(V_{3,3}\right)$ is defined by

$$
\begin{cases}e_{A} e_{B}=(-1)^{n((A \cap B) \backslash N)}(-1)^{P(A, B)} e_{A \triangle B}, & \text { if } A, B \in \mathcal{P} N, \\ \lambda \mu=\sum_{A, B \in \mathcal{P} N} \lambda_{A} \mu_{B} e_{A} e_{B}, & \text { if } \lambda=\sum_{A \in \mathcal{P} N} \lambda_{A} e_{A}, \\ & \mu=\sum_{B \in \mathcal{P} N} \mu_{B} e_{B},\end{cases}
$$

where $n(A)$ is the cardinal number of the set $A$, the number $P(A, B)=\sum_{j \in B} P(A, j), P(A, j)=$ $n\{i, i \in A, i>j\}$, the symmetric difference set $A \Delta B$ is order-preserving in the above way, and $\lambda_{A} \in \mathbb{R}$ is the coefficient of the $e_{A}$-component of the Clifford number $\lambda$. It follows from the multiplication rule above that $e_{0}$ is the identity element written now as 1 and, in particular,

$$
\begin{cases}e_{i}^{2}=1, & \text { if } i=1,2,3 \\ e_{i} e_{j}=-e_{j} e_{i}, & \text { if } 1 \leq i<j \leq 3 .\end{cases}
$$

Thus $C l\left(V_{3,3}\right)$ is a real linear, associative, but non-commutative algebra. An involution is defined by

$$
\begin{cases}\overline{e_{A}}=(-1)^{\frac{n(A)(n(A)+3)}{2}} e_{A}, & \text { if } A \in \mathcal{P} N, \\ \bar{\lambda}=\sum_{A \in \mathcal{P} N} \lambda_{A} \overline{e_{A}}, & \text { if } \lambda=\sum_{A \in \mathcal{P} N} \lambda_{A} e_{A} .\end{cases}
$$

In view of the multiplication rule (2.1) and the definition of the involution (2.3), it is easy to check that

$$
\begin{cases}\overline{e_{i}}=e_{i}, & \text { if } i=0,1,2,3 \\ \overline{\lambda \mu}=\bar{\mu} \bar{\lambda}, & \text { for any } \lambda, \mu \in \operatorname{Cl}\left(V_{3,3}\right) .\end{cases}
$$


The norm of $\lambda$ is defined by $\|\lambda\|=\left(\sum_{A \in \mathcal{P} N}\left|\lambda_{A}\right|^{2}\right)^{\frac{1}{2}}$. Throughout this article, suppose $\Omega$ is an open bounded non-empty subset of $\mathbb{R}^{3}$ with a Lyapunov boundary $\partial \Omega$, denote $\Omega^{+}=\Omega, \Omega^{-}=\mathbb{R}^{3} \backslash \bar{\Omega}$. We now introduce the Dirac operator $D=\sum_{i=1}^{3} e_{i} \frac{\partial}{\partial x_{i}}$. In particular, we have $D D=\Delta$ where $\Delta$ is the Laplacian over $\mathbb{R}^{3}$. A function $u: \Omega \mapsto C l\left(V_{3,3}\right)$ is said to be left monogenic if it satisfies the equation $D[u](\mathbf{x})=0$ for each $\mathbf{x} \in \Omega$. A similar definition can be given for right monogenic functions. Elementary properties of the Dirac operators and left monogenic functions can be found in [8, 9, 26-29].

The elliptic partial differential operator $H=\left(\Delta-\kappa^{2}\right)$, for $\kappa>0$, corresponds to the modified Helmholtz equation:

$$
H u=\left(\Delta-\kappa^{2}\right) u=0
$$

which has as fundamental solution the function

$$
E_{1}\left(\mathbf{x}, \kappa^{2}\right)=\frac{e^{-\kappa\|\mathbf{x}\|}}{4 \pi\|\mathbf{x}\|}
$$

We defined the operators $L_{\kappa}, L_{-\kappa}$ as follows:

$$
L_{\kappa} u=D u+\kappa u, \quad L_{-\kappa} u=D u-\kappa u .
$$

By the multiplication rule on Clifford algebra $C l\left(V_{3,3}\right)$, the modified Helmholtz equation may be written as

$$
L_{\kappa} L_{-\kappa} u=L_{-\kappa} L_{\kappa} u=0 .
$$

Denote

$$
\begin{aligned}
& K_{1}(\mathbf{x}, \mathbf{y}, \kappa)=\frac{1}{4 \pi}\left(\frac{\mathbf{y}-\mathbf{x}}{\|\mathbf{y}-\mathbf{x}\|^{3}}+\frac{\kappa(\mathbf{y}-\mathbf{x})}{\|\mathbf{y}-\mathbf{x}\|^{2}}+\frac{\kappa}{\|\mathbf{y}-\mathbf{x}\|}\right) e^{-\kappa\|\mathbf{y}-\mathbf{x}\|}, \\
& K_{* 1}(\mathbf{x}, \mathbf{y}, \kappa)=\frac{1}{4 \pi}\left(\frac{\mathbf{y}-\mathbf{x}}{\|\mathbf{y}-\mathbf{x}\|^{3}}+\frac{\kappa(\mathbf{y}-\mathbf{x})}{\|\mathbf{y}-\mathbf{x}\|^{2}}-\frac{\kappa}{\|\mathbf{y}-\mathbf{x}\|}\right) e^{-\kappa\|\mathbf{y}-\mathbf{x}\|},
\end{aligned}
$$

where $\mathbf{y}-\mathbf{x}=\sum_{i=1}^{3}\left(y_{i}-x_{i}\right) e_{i}$. It is clear that $K_{1}(\mathbf{x}, \mathbf{y}, \kappa)$ and $K_{* 1}(\mathbf{x}, \mathbf{y}, \kappa)$ are fundamental solutions of $L_{\kappa}=\sum_{i=1}^{3} e_{i} \frac{\partial}{\partial y_{i}}+\kappa$ and $L_{-\kappa}=\sum_{i=1}^{3} e_{i} \frac{\partial}{\partial y_{i}}-\kappa$, respectively.

\section{Integral representation formulas and some properties of generalized Cauchy integral operators}

Let $\Omega$ be an open bounded nonempty subset of $\mathbb{R}^{3}$ with a Lyapunov boundary $\partial \Omega, u(\mathbf{x})=$ $\sum_{A} e_{A} u_{A}(\mathbf{x})$, where $u_{A}(\mathbf{x})$ are real functions. $u(\mathbf{x})$ is called a Hölder continuous functions on $\bar{\Omega}$ if the following condition is satisfied:

$$
\left\|u\left(\mathbf{x}_{1}\right)-u\left(\mathbf{x}_{2}\right)\right\|=\left[\sum_{A}\left\|u_{A}\left(\mathbf{x}_{1}\right)-u_{A}\left(\mathbf{x}_{2}\right)\right\|\right]^{\frac{1}{2}} \leq C\left\|\mathbf{x}_{1}-\mathbf{x}_{2}\right\|^{\alpha},
$$

where for any $\mathbf{x}_{1}, \mathbf{x}_{2} \in \bar{\Omega}, \mathbf{x}_{1} \neq \mathbf{x}_{2}, 0<\alpha \leq 1, C$ is a positive constant independent of $\mathbf{x}_{1}, \mathbf{x}_{2}$. 
Denote by $H^{\alpha}\left(\partial \Omega, C l\left(V_{3,3}\right)\right)$ the set of Hölder continuous functions with values in $C l\left(V_{3,3}\right)$ on $\partial \Omega$ (the Hölder exponent is $\left.\alpha, 0<\alpha<1\right)$. Define the norm of $u$ in $H^{\alpha}(\partial \Omega$, $\left.\mathrm{Cl}\left(V_{3,3}\right)\right)$ as

$$
\|u\|_{(\alpha, \partial \Omega)}=\|u\|_{\infty}+\|u\|_{\alpha}
$$

where $\|u\|_{\infty}:=\sup _{\mathbf{x} \in \partial \Omega}\|u(\mathbf{x})\|,\|u\|_{\alpha}:=\sup _{\substack{\mathbf{x}_{1}, \mathbf{x}_{2} \in \partial \Omega \\ \mathbf{x}_{1} \neq \mathbf{x}_{2}}} \frac{\left\|u\left(\mathbf{x}_{1}\right)-u\left(\mathbf{x}_{2}\right)\right\|}{\left\|\mathbf{x}_{1}-\mathbf{x}_{2}\right\|^{\alpha}}$

Lemma 3.1 [22] The Hölder space $H^{\alpha}\left(\partial \Omega, C l\left(V_{3,3}\right)\right)$ is a Banach space with norm (3.1).

Lemma 3.2 Let $f, g \in C^{1}\left(\Omega, C l\left(V_{3,3}\right)\right) \cap C\left(\bar{\Omega}, C l\left(V_{3,3}\right)\right)$. Then

$$
\int_{\partial \Omega} f d \sigma_{\mathrm{y}} g=\int_{\Omega}[f] L_{\kappa} g d V+\int_{\Omega} f L_{-\kappa}[g] d V=\int_{\Omega}[f] L_{-\kappa} g d V+\int_{\Omega} f L_{\kappa}[g] d V .
$$

Proof From Stokes' theorem in Clifford analysis in [26], the results can be directly proved.

Theorem 3.3 If $u \in C^{2}\left(\Omega, C l\left(V_{3,3}\right)\right) \cap C^{1}\left(\bar{\Omega}, C l\left(V_{3,3}\right)\right)$ where $\Omega$ is an open bounded nonempty subset of $\mathbb{R}^{3}$ with a Lyapunov boundary $\partial \Omega$, then

$$
\begin{gathered}
\int_{\partial \Omega} K_{* 1}(\mathbf{x}, \mathbf{y}, \kappa) d \sigma_{\mathbf{y}} u(\mathbf{y})+\frac{1}{4 \pi} \int_{\partial \Omega} \frac{e^{-\kappa\|\mathbf{y}-\mathbf{x}\|}}{\|\mathbf{y}-\mathbf{x}\|} d \sigma_{\mathbf{y}} L_{\kappa}[u](\mathbf{y}) \\
-\frac{1}{4 \pi} \int_{\Omega} \frac{e^{-\kappa\|\mathbf{y}-\mathbf{x}\|}}{\|\mathbf{y}-\mathbf{x}\|} H[u](\mathbf{y}) d V= \begin{cases}u(\mathbf{x}), & \mathbf{x} \in \Omega, \\
0, & \mathbf{x} \in \mathbb{R}^{3} \backslash \bar{\Omega},\end{cases}
\end{gathered}
$$

where $K_{* 1}(\mathbf{x}, \mathbf{y}, \kappa)$ is as in $(2.8)$.

Proof Let $\mathbf{x} \in \mathbb{R}^{3} \backslash \bar{\Omega}$. Using Lemma 3.2, we get

$$
\begin{aligned}
& \frac{1}{4 \pi} \int_{\Omega} \frac{e^{-\kappa\|\mathbf{y}-\mathbf{x}\|}}{\|\mathbf{y}-\mathbf{x}\|} H[u](\mathbf{y}) d V \\
& \quad=\frac{1}{4 \pi} \int_{\partial \Omega} \frac{e^{-\kappa\|\mathbf{y}-\mathbf{x}\|}}{\|\mathbf{y}-\mathbf{x}\|} d \sigma_{\mathbf{y}} L_{\kappa}[u](\mathbf{y})-\frac{1}{4 \pi} \int_{\Omega}\left[\frac{e^{-\kappa\|\mathbf{y}-\mathbf{x}\|}}{\|\mathbf{y}-\mathbf{x}\|}\right] L_{\kappa} L_{\kappa}[u](\mathbf{y}) d V \\
& \quad=\frac{1}{4 \pi} \int_{\partial \Omega} \frac{e^{-\kappa\|\mathbf{y}-\mathbf{x}\|}}{\|\mathbf{y}-\mathbf{x}\|} d \sigma_{\mathbf{y}} L_{\kappa}[u](\mathbf{y})+\int_{\Omega} K_{* 1}(\mathbf{x}, \mathbf{y}, \kappa) L_{\kappa}[u](\mathbf{y}) d V \\
& \quad=\frac{1}{4 \pi} \int_{\partial \Omega} \frac{e^{-\kappa\|\mathbf{y}-\mathbf{x}\|}}{\|\mathbf{y}-\mathbf{x}\|} d \sigma_{\mathbf{y}} L_{\kappa}[u](\mathbf{y})+\int_{\partial \Omega} K_{* 1}(\mathbf{x}, \mathbf{y}, \kappa) d \sigma_{\mathbf{y}} u(\mathbf{y}) .
\end{aligned}
$$

Then the left-hand side of (3.2) apparently equals zero.

Now, let $\mathbf{x} \in \Omega$ and take $r>0$ such that $B(\mathbf{x}, r) \subset \Omega$. Invoking the previous case, we may then write

$$
\begin{aligned}
& \int_{\partial(\Omega \backslash B(\mathbf{x}, r))} K_{* 1}(\mathbf{x}, \mathbf{y}, \kappa) d \sigma_{\mathbf{y}} u(\mathbf{y})+\frac{1}{4 \pi} \int_{\partial(\Omega \backslash B(\mathbf{x}, r))} \frac{e^{-\kappa\|\mathbf{y}-\mathbf{x}\|}}{\|\mathbf{y}-\mathbf{y}\|} d \sigma_{\mathbf{y}} L_{\kappa}[u](\mathbf{y}) \\
& -\frac{1}{4 \pi} \int_{\Omega \backslash B(\mathbf{x}, r)} \frac{e^{-\kappa\|\mathbf{y}-\mathbf{x}\|}}{\|\mathbf{y}-\mathbf{y}\|} H[u](\mathbf{y}) d V=0 .
\end{aligned}
$$


Here we take the limits for $r \rightarrow 0$. As regards the weak singularity of $\frac{e^{-\kappa\|y-x\|}}{\|\mathbf{y}-\mathbf{x}\|}$, the third term of (3.3) yields

$$
\lim _{r \rightarrow 0} \int_{\Omega \backslash B(\mathbf{x}, r)} \frac{e^{-\kappa\|\mathbf{y}-\mathbf{x}\|}}{\|\mathbf{y}-\mathbf{x}\|} H[u](\mathbf{y}) d V=\int_{\Omega} \frac{e^{-\kappa\|\mathbf{y}-\mathbf{x}\|}}{\|\mathbf{y}-\mathbf{x}\|} H[u](\mathbf{y}) d V .
$$

Furthermore we write

$$
\begin{aligned}
& \int_{\partial(\Omega \backslash B(\mathbf{x}, r))} K_{* 1}(\mathbf{x}, \mathbf{y}, \kappa) d \sigma_{\mathbf{y}} u(\mathbf{y})+\frac{1}{4 \pi} \int_{\partial(\Omega \backslash B(\mathbf{x}, r))} \frac{e^{-\kappa\|\mathbf{y}-\mathbf{x}\|}}{\|\mathbf{y}-\mathbf{x}\|} d \sigma_{\mathbf{y}} L_{\kappa}[u](\mathbf{y}) \\
& =\int_{\partial \Omega} K_{* 1}(\mathbf{x}, \mathbf{y}, \kappa) d \sigma_{\mathbf{y}} u(\mathbf{y})+\frac{1}{4 \pi} \int_{\partial \Omega} \frac{e^{-\kappa\|\mathbf{y}-\mathbf{x}\|}}{\|\mathbf{y}-\mathbf{x}\|} d \sigma_{\mathbf{y}} L_{\kappa}[u](\mathbf{y}) \\
& \quad-\int_{\partial B(\mathbf{x}, r)} K_{* 1}(\mathbf{x}, \mathbf{y}, \kappa) d \sigma_{\mathbf{y}} u(\mathbf{y})-\frac{1}{4 \pi} \int_{\partial B(\mathbf{x}, r)} \frac{e^{-\kappa\|\mathbf{y}-\mathbf{x}\|}}{\|\mathbf{y}-\mathbf{x}\|} d \sigma_{\mathbf{y}} L_{\kappa}[u](\mathbf{y}) .
\end{aligned}
$$

We denote

$$
\Theta(\mathbf{x}) \triangleq \int_{\partial B(\mathbf{x}, r)} K_{* 1}(\mathbf{x}, \mathbf{y}, \kappa) d \sigma_{\mathbf{y}} u(\mathbf{y})+\frac{1}{4 \pi} \int_{\partial B(\mathbf{x}, r)} \frac{e^{-\kappa\|\mathbf{y}-\mathbf{x}\|}}{\|\mathbf{y}-\mathbf{x}\|} d \sigma_{\mathbf{y}} L_{\kappa}[u](\mathbf{y}) .
$$

It follows from the Stokes formula that

$$
\begin{aligned}
\Theta(\mathbf{x})= & \frac{3 e^{-\kappa r}}{4 \pi r^{3}} \int_{B(\mathbf{x}, r)} u(\mathbf{y}) d V+\frac{3 \kappa e^{-\kappa r}}{4 \pi r^{2}} \int_{B(\mathbf{x}, r)} u(\mathbf{y}) d V \\
& +\frac{e^{-\kappa r}}{4 \pi r^{3}} \int_{B(\mathbf{x}, r)}(\mathbf{y}-\mathbf{x}) D[u](\mathbf{y}) d V+\frac{\kappa e^{-\kappa r}}{4 \pi r^{2}} \int_{B(\mathbf{x}, r)}(\mathbf{y}-\mathbf{x}) D[u](\mathbf{y}) d V \\
& +\frac{e^{-\kappa r}}{4 \pi r} \int_{B(\mathbf{x}, r)} \Delta[u](\mathbf{y}) d V .
\end{aligned}
$$

Applying the Lebesgue differentiation theorem, we have

$$
\lim _{r \rightarrow 0} \Theta(\mathbf{x})=u(\mathbf{x})
$$

Combining (3.3) with (3.4)-(3.8), we get the desired result.

Theorem 3.4 If $u \in C^{2}\left(\Omega, C l\left(V_{3,3}\right)\right) \cap C^{1}\left(\bar{\Omega}, C l\left(V_{3,3}\right)\right)$ where $\Omega$ is an open bounded nonempty subset of $\mathbb{R}^{3}$ with a Lyapunov boundary $\partial \Omega$, then

$$
\begin{gathered}
\int_{\partial \Omega} K_{1}(\mathbf{x}, \mathbf{y}, \kappa) d \sigma_{\mathbf{y}} u(\mathbf{y})+\frac{1}{4 \pi} \int_{\partial \Omega} \frac{e^{-\kappa\|\mathbf{y}-\mathbf{x}\|}}{\|\mathbf{y}-\mathbf{x}\|} d \sigma_{\mathbf{y}} L_{-\kappa}[u](\mathbf{y}) \\
-\frac{1}{4 \pi} \int_{\Omega} \frac{e^{-\kappa\|\mathbf{y}-\mathbf{x}\|}}{\|\mathbf{y}-\mathbf{x}\|} H[u](\mathbf{y}) d V= \begin{cases}u(\mathbf{x}), & \mathbf{x} \in \Omega, \\
0, & \mathbf{x} \in \mathbb{R}^{3} \backslash \bar{\Omega},\end{cases}
\end{gathered}
$$

where $K_{1}(\mathbf{x}, \mathbf{y}, \kappa)$ is as in (2.7).

Proof The result can be similarly proved to Theorem 3.3. 
Corollary 3.5 If $u \in C^{2}\left(\Omega, C l\left(V_{3,3}\right)\right) \cap C^{1}\left(\bar{\Omega}, C l\left(V_{3,3}\right)\right)$ where $\Omega$ is an open bounded nonempty subset of $\mathbb{R}^{3}$ with a Lyapunov boundary $\partial \Omega$ and $H[u]=L_{\kappa} L_{-\kappa}[u]=0$ in $\Omega$, then

$$
\int_{\partial \Omega} K_{1}(\mathbf{x}, \mathbf{y}, \kappa) d \sigma_{y} u(\mathbf{y})+\frac{1}{4 \pi} \int_{\partial \Omega} \frac{e^{-\kappa\|\mathbf{y}-\mathbf{x}\|}}{\|\mathbf{y}-\mathbf{x}\|} d \sigma_{\mathbf{y}} L_{-\kappa}[u](\mathbf{y})= \begin{cases}u(\mathbf{x}), & \mathbf{x} \in \Omega \\ 0, & \mathbf{x} \in \mathbb{R}^{3} \backslash \bar{\Omega}\end{cases}
$$

where $K_{1}(\mathbf{x}, \mathbf{y}, \kappa)$ is as in (2.7).

Corollary 3.6 If $u \in C^{2}\left(\Omega, C l\left(V_{3,3}\right)\right) \cap C^{1}\left(\bar{\Omega}, C l\left(V_{3,3}\right)\right)$ where $\Omega$ is an open bounded nonempty subset of $\mathbb{R}^{3}$ with a Lyapunov boundary $\partial \Omega$ and $H[u]=L_{-\kappa} L_{\kappa}[u]=0$ in $\Omega$, then

$$
\int_{\partial \Omega} K_{* 1}(\mathbf{x}, \mathbf{y}, \kappa) d \sigma_{y} u(\mathbf{y})+\frac{1}{4 \pi} \int_{\partial \Omega} \frac{e^{-\kappa\|\mathbf{y}-\mathbf{x}\|}}{\|\mathbf{y}-\mathbf{x}\|} d \sigma_{\mathbf{y}} L_{\kappa}[u](\mathbf{y})= \begin{cases}u(\mathbf{x}), & \mathbf{x} \in \Omega, \\ 0, & \mathbf{x} \in \mathbb{R}^{3} \backslash \bar{\Omega},\end{cases}
$$

where $K_{* 1}(\mathbf{x}, \mathbf{y}, \kappa)$ is as in $(2.8)$.

Corollary 3.7 Let $f(\mathbf{x}) \in C_{c}^{2}\left(\Omega, C l\left(V_{3,3}\right)\right)$. The solution of the following Dirichlet boundary value problem:

$$
\begin{cases}H u=f, & \text { in } \Omega, \\ L_{\kappa}[u]=0, & \text { on } \partial \Omega, \\ u=0, & \text { on } \partial \Omega,\end{cases}
$$

is

$$
u(\mathbf{x})=-\frac{1}{4 \pi} \int_{\Omega} \frac{e^{-\kappa\|\mathbf{y}-\mathbf{x}\|}}{\|\mathbf{y}-\mathbf{x}\|} f(\mathbf{y}) d V .
$$

Proof By Theorem 3.3, the solution of (3.12) is formulated as

$$
\begin{aligned}
u(\mathbf{x})= & \int_{\partial \Omega} K_{* 1}(\mathbf{x}, \mathbf{y}, \kappa) d \sigma_{\mathbf{y}} u(\mathbf{y})+\frac{1}{4 \pi} \int_{\partial \Omega} \frac{e^{-\kappa\|\mathbf{y}-\mathbf{x}\|}}{\|\mathbf{y}-\mathbf{x}\|} d \sigma_{\mathbf{y}} L_{\kappa}[u](\mathbf{y}) \\
& -\frac{1}{4 \pi} \int_{\Omega} \frac{e^{-\kappa\|\mathbf{y}-\mathbf{x}\|}}{\|\mathbf{y}-\mathbf{x}\|} H[u](\mathbf{y}) d V,
\end{aligned}
$$

since $L_{\kappa}[u]=0$ and $u=0$ on $\partial \Omega$, the result follows.

Using Theorem 3.4, we also have the following result which can be similarly proved to Corollary 3.7.

Corollary 3.8 Let $f(\mathbf{x}) \in C_{c}^{2}\left(\Omega, C l\left(V_{3,3}\right)\right)$. The solution of the Dirichlet boundary value problem

$$
\begin{cases}H u=f, & \text { in } \Omega, \\ L_{-\kappa}[u]=0, & \text { on } \partial \Omega, \\ u=0, & \text { on } \partial \Omega,\end{cases}
$$

is

$$
u(\mathbf{x})=-\frac{1}{4 \pi} \int_{\Omega} \frac{e^{-\kappa\|\mathbf{y}-\mathbf{x}\|}}{\|\mathbf{y}-\mathbf{x}\|} f(\mathbf{y}) d V .
$$


Next, we introduce the following generalized Teodorescu operators $\mathbb{T}_{ \pm \kappa}$, the generalized Cauchy integral operators $\mathbb{F}_{ \pm \kappa}$, and the generalized Cauchy singular integral operators $\mathbb{S}_{ \pm \kappa}$ :

$$
\begin{aligned}
& \mathbb{T}_{\kappa}[u](\mathbf{x}) \triangleq-\int_{\Omega} K_{* 1}(\mathbf{x}, \mathbf{y}, \kappa) u(\mathbf{y}) d V, \quad \mathbf{x} \in \Omega, \\
& \mathbb{T}_{-\kappa}[u](\mathbf{x}) \triangleq-\int_{\Omega} K_{1}(\mathbf{x}, \mathbf{y}, \kappa) u(\mathbf{y}) d V, \quad \mathbf{x} \in \Omega, \\
& \mathbb{F}_{\kappa}[u](\mathbf{x}) \triangleq \int_{\partial \Omega} K_{* 1}(\mathbf{x}, \mathbf{y}, \kappa) d \sigma_{y} u(\mathbf{y}), \quad \mathbf{x} \in \mathbb{R}^{3} \backslash \partial \Omega, \\
& \mathbb{F}_{-\kappa}[u](\mathbf{x}) \triangleq \int_{\partial \Omega} K_{1}(\mathbf{x}, \mathbf{y}, \kappa) d \sigma_{y} u(\mathbf{y}), \quad \mathbf{x} \in \mathbb{R}^{3} \backslash \partial \Omega, \\
& \mathbb{S}_{\kappa}[u](\mathbf{x}) \triangleq 2 \int_{\partial \Omega} K_{* 1}(\mathbf{x}, \mathbf{y}, \kappa) d \sigma_{y} u(\mathbf{y}), \quad \mathbf{x} \in \partial \Omega, \\
& \mathbb{S}_{-\kappa}[u](\mathbf{x}) \triangleq 2 \int_{\partial \Omega} K_{1}(\mathbf{x}, \mathbf{y}, \kappa) d \sigma_{y} u(\mathbf{y}), \quad \mathbf{x} \in \partial \Omega,
\end{aligned}
$$

where $\kappa \geq 0, u \in H^{\alpha}\left(\partial \Omega, C l\left(V_{3,3}\right)\right)$.

Lemma 3.9 [22] Let $\Omega$ be an open nonempty bounded subset of $\mathbb{R}^{3}$ with a Lyapunov boundary $\partial \Omega, u \in H^{\alpha}\left(\partial \Omega, C l\left(V_{3,3}\right)\right), 0<\alpha \leq 1$. Then

$$
\begin{aligned}
& \lim _{\substack{\mathbf{x} \rightarrow \mathbf{x}_{0} \in \partial \Omega \\
\mathbf{x} \in \Omega}} \mathbb{F}_{\kappa}[u](\mathbf{x})=\frac{u\left(\mathbf{x}_{0}\right)}{2}+\frac{1}{2} \mathbb{S}_{\kappa}[u]\left(\mathbf{x}_{0}\right), \\
& \lim _{\substack{\mathbf{x} \rightarrow \mathbf{x}_{0} \in \partial \Omega \\
\mathbf{x} \in \mathbb{R}^{3} \backslash \bar{\Omega}}} \mathbb{F}_{\kappa}[u](\mathbf{x})=-\frac{u\left(\mathbf{x}_{0}\right)}{2}+\frac{1}{2} \mathbb{S}_{\kappa}[u]\left(\mathbf{x}_{0}\right), \\
& \lim _{\substack{\mathbf{x} \rightarrow \mathbf{x}_{0} \in \partial \Omega \\
\mathbf{x} \in \Omega}} \mathbb{F}_{-\kappa}[u](\mathbf{x})=\frac{u\left(\mathbf{x}_{0}\right)}{2}+\frac{1}{2} \mathbb{S}_{-\kappa}[u]\left(\mathbf{x}_{0}\right), \\
& \lim _{\substack{\mathbf{x} \rightarrow \mathbf{x}_{0} \in \partial \Omega \\
\mathbf{x} \in \mathbb{R}^{3} \backslash \bar{\Omega}}} \mathbb{F}_{-\kappa}[u](\mathbf{x})=-\frac{u\left(\mathbf{x}_{0}\right)}{2}+\frac{1}{2} \mathbb{S}_{-\kappa}[u]\left(\mathbf{x}_{0}\right) .
\end{aligned}
$$

Theorem 3.10 Let $\Omega$ be an open bounded non-empty subset of $\mathbb{R}^{3}$ with a Lyapunov boundary $\partial \Omega, u \in C^{1}\left(\Omega, C l\left(V_{3,3}\right)\right) \cap C\left(\bar{\Omega}, C l\left(V_{3,3}\right)\right)$. Then for $\mathbf{x} \in \Omega$,

$$
L_{\kappa} \mathbb{T}_{\kappa}[u](\mathbf{x})=u(\mathbf{x})
$$

Proof Step 1. Because $u(\mathbf{x})$ has its compact support supp $[u] \Subset \Omega$, we have

$$
\begin{aligned}
\mathbb{T}_{\kappa}[u](\mathbf{x}) & =-\int_{\Omega} K_{* 1}(\mathbf{x}, \mathbf{y}, \kappa) u(\mathbf{y}) d V \\
& =-\int_{\mathbb{R}^{3}} K_{* 1}(\mathbf{x}, \mathbf{y}, \kappa) u(\mathbf{y}) d V \\
& =-\int_{\mathbb{R}^{3}} K_{* 1}(\mathbf{x}, \mathbf{y}+\mathbf{x}, \kappa) u(\mathbf{y}+\mathbf{x}) d V .
\end{aligned}
$$


In view of $u(\mathbf{x})$ having a compact support, the operator $L_{\kappa}$ acting on $\mathbb{T}_{\kappa}[u](\mathbf{x})$ may be interchanged with integration. Thus we get

$$
\begin{aligned}
L_{\kappa} \mathbb{T}_{\kappa}[u](\mathbf{x})= & -\lim _{r \rightarrow 0} \int_{\mathbb{R}^{3} \backslash B(0, r)}\left[\sum_{i=1}^{3} e_{i} \frac{\partial}{\partial x_{i}}\left[K_{* 1}(\mathbf{x}, \mathbf{y}+\mathbf{x}, \kappa) u(\mathbf{y}+\mathbf{x})\right]\right. \\
& \left.+\kappa K_{* 1}(\mathbf{x}, \mathbf{y}+\mathbf{x}, \kappa) u(\mathbf{y}+\mathbf{x})\right] d V \\
= & -\lim _{r \rightarrow 0} \int_{\mathbb{R}^{3} \backslash B(0, r)}\left[\sum_{i=1}^{3} e_{i} K_{* 1}(\mathbf{x}, \mathbf{y}+\mathbf{x}, \kappa) \frac{\partial}{\partial x_{i}} u(\mathbf{y}+\mathbf{x})\right. \\
& \left.+\kappa K_{* 1}(\mathbf{x}, \mathbf{y}+\mathbf{x}, \kappa) u(\mathbf{y}+\mathbf{x})\right] d V \\
= & -\lim _{r \rightarrow 0} \int_{\mathbb{R}^{3} \backslash B(0, r)}\left[\sum_{i=1}^{3} e_{i} K_{* 1}(\mathbf{x}, \mathbf{y}+\mathbf{x}, \kappa) \frac{\partial}{\partial y_{i}} u(\mathbf{y}+\mathbf{x})\right. \\
& \left.+\kappa K_{* 1}(\mathbf{x}, \mathbf{y}+\mathbf{x}, \kappa) u(\mathbf{y}+\mathbf{x})\right] d V \\
= & -\lim _{r \rightarrow 0} \int_{\mathbb{R}^{3} \backslash B(0, r)}\left[\sum_{i=1}^{3} e_{i} \frac{\partial}{\partial y_{i}}\left[K_{* 1}(\mathbf{x}, \mathbf{y}+\mathbf{x}, \kappa) u(\mathbf{y}+\mathbf{x})\right]\right] d V .
\end{aligned}
$$

Using the Stokes formula, we conclude that

$$
\begin{aligned}
L_{\kappa} \mathbb{T}_{\kappa}[u](\mathbf{x})= & \lim _{r \rightarrow 0} \int_{\|\mathbf{y}\|=r} d \sigma_{\mathbf{y}} K_{* 1}(\mathbf{x}, \mathbf{y}+\mathbf{x}, \kappa) u(\mathbf{y}+\mathbf{x}) \\
= & \lim _{r \rightarrow 0} \int_{\|\mathbf{y}\|=r} d \sigma_{\mathbf{y}} K_{* 1}(\mathbf{x}, \mathbf{y}+\mathbf{x}, \kappa)[u(\mathbf{y}+\mathbf{x})-u(\mathbf{x})] \\
& +\lim _{r \rightarrow 0} \int_{\|\mathbf{y}\|=r} d \sigma_{\mathbf{y}} K_{* 1}(\mathbf{x}, \mathbf{y}+\mathbf{x}, \kappa) u(\mathbf{x}) \\
= & \lim _{r \rightarrow 0} \frac{1}{4 \pi} \int_{\|\mathbf{y}\|=r} d \sigma_{\mathbf{y}}\left(\frac{\mathbf{y}}{\|\mathbf{y}\|^{3}}+\frac{\kappa \mathbf{y}}{\|\mathbf{y}\|^{2}}-\frac{\kappa}{\|\mathbf{y}\|}\right) e^{-\kappa\|\mathbf{y}\|} u(\mathbf{x}) \\
= & \lim _{r \rightarrow 0}\left(\frac{3 e^{-\kappa r}}{4 \pi r^{3}} \int_{\|\mathbf{y}\| \leq r} d V+\frac{3 \kappa e^{-\kappa r}}{4 \pi r^{2}} \int_{\|\mathbf{y}\| \leq r} d V\right) u(\mathbf{x}) \\
= & u(\mathbf{x}) .
\end{aligned}
$$

Thus we have proved that (3.27) follows for any $u(\mathbf{x}) \in C_{c}^{1}\left(\Omega, C l\left(V_{3,3}\right)\right)$.

Step 2. We prove that (3.27) holds for any $u(\mathbf{x}) \in C^{1}\left(\Omega, C l\left(V_{3,3}\right)\right)$. We take a neighborhood $V$ of $\mathbf{x}$ such that $\mathbf{x} \in V \Subset \Omega$, a real-valued function $\Psi \in C^{\infty}(\Omega)$ such that $\left.\Psi\right|_{V}=1$ and $\operatorname{supp} \Psi \Subset \Omega$. Then

$$
u(\mathbf{x})=u \Psi+u(1-\Psi):=u_{1}(\mathbf{x})+u_{2}(\mathbf{x})
$$

It is obvious that $u_{1}(\mathbf{x}) \in C_{c}^{1}\left(\Omega, C l\left(V_{3,3}\right)\right), u_{2}(\mathbf{x}) \in C^{1}\left(\Omega, C l\left(V_{3,3}\right)\right)$ and $\left.u_{1}\right|_{V}=u,\left.u_{2}\right|_{V}=0$. Following step 1, we obtain

$$
L_{\kappa} \mathbb{T}_{\kappa}\left[u_{1}\right](\mathbf{x})=u_{1}(\mathbf{x})=u(\mathbf{x}), \quad \mathbf{x} \in V .
$$


Since $u_{2}(\mathbf{x})$ equals zero in $V$, we get

$$
\begin{aligned}
L_{\kappa} \mathbb{T}_{\kappa}\left[u_{2}\right](\mathbf{x}) & =L_{\kappa}\left[-\int_{\Omega} K_{* 1}(\mathbf{x}, \mathbf{y}, \kappa) u_{2}(\mathbf{y}) d V\right] \\
& =L_{\kappa}\left[-\int_{\Omega \backslash V} K_{* 1}(\mathbf{x}, \mathbf{y}, \kappa) u_{2}(\mathbf{y}) d V\right] \\
& =0
\end{aligned}
$$

It follows from (3.29) and (3.30) that

$$
L_{\kappa} \mathbb{T}_{\kappa}[u](\mathbf{x})=u(\mathbf{x})
$$

Because $\mathbf{x}$ is taken arbitrarily in $\Omega$, the result follows.

Corresponding to Theorem 3.10, we have the following theorem.

Theorem 3.11 Let $\Omega$ be an open bounded non-empty subset of $\mathbb{R}^{3}$ with a Lyapunov boundary $\partial \Omega, u \in C^{1}\left(\Omega, C l\left(v_{3,3}\right)\right) \cap C\left(\bar{\Omega}, C l\left(V_{3,3}\right)\right)$. Then for $\mathbf{x} \in \Omega$,

$$
L_{-\kappa} \mathbb{T}_{-\kappa}[u](\mathbf{x})=u(\mathbf{x})
$$

In the following, we need to consider Hölder's boundedness of the singular integral operators $\mathbb{S}_{ \pm \kappa}$. It is necessary to solve the following boundary value problems in Clifford analysis.

Theorem 3.12 Let $\Omega$ be an open nonempty bounded subset of $\mathbb{R}^{3}$ with a Lyapunov boundary $\partial \Omega$. Then the generalized Cauchy integral operator $\mathbb{S}_{\kappa}: H^{\alpha}\left(\partial \Omega, C l\left(V_{3,3}\right)\right) \mapsto$ $H^{\alpha}\left(\partial \Omega, C l\left(V_{3,3}\right)\right)$ defined by (3.21) is bounded, i.e.

$$
\left\|\mathbb{S}_{\kappa}[u]\right\|_{(\alpha, \partial \Omega)} \leq C\|u\|_{(\alpha, \partial \Omega)}
$$

where $C=\max \left\{\frac{C_{4}}{2 \pi} \eta^{2-\alpha}\left(|\partial \Omega|+\frac{1}{2-\alpha}\right)+C_{6}, \frac{C_{5}\left(|\partial \Omega|+\eta^{2}+\eta\right)}{2 \pi \eta}+\frac{2 \kappa\left(C_{2} \eta^{2}+C_{3}|\partial \Omega|\right)}{\eta}\right\}$ and $|\partial \Omega|$ denotes the surface area of $\Omega$.

Proof For $\mathbf{x} \in \partial \Omega$, we have

$$
\begin{aligned}
\left\|\mathbb{S}_{\kappa}[u](\mathbf{x})\right\|= & \left\|\frac{2}{4 \pi} \int_{\partial \Omega}\left[\frac{\mathbf{y}-\mathbf{x}}{\|\mathbf{y}-\mathbf{x}\|^{3}}+\frac{\kappa(\mathbf{y}-\mathbf{x})}{\|\mathbf{y}-\mathbf{x}\|^{2}}-\frac{\kappa}{\|\mathbf{y}-\mathbf{x}\|}\right] e^{-\kappa\|\mathbf{y}-\mathbf{x}\|} d \sigma_{\mathbf{y}} u(\mathbf{y})\right\| \\
\leq & \left\|\frac{1}{2 \pi} \int_{\partial \Omega} \frac{\kappa(\mathbf{y}-\mathbf{x})}{\|\mathbf{y}-\mathbf{x}\|^{2}} e^{-\kappa\|\mathbf{y}-\mathbf{x}\|} d \sigma_{\mathbf{y}} u(\mathbf{y})\right\| \\
& +\left\|\frac{1}{2 \pi} \int_{\partial \Omega} \frac{\kappa}{\|\mathbf{y}-\mathbf{x}\|} e^{-\kappa\|\mathbf{y}-\mathbf{x}\|} d \sigma_{\mathbf{y}} u(\mathbf{y})\right\| \\
& +\left\|\frac{1}{2 \pi} \int_{\partial \Omega} \frac{\mathbf{y}-\mathbf{x}}{\|\mathbf{y}-\mathbf{x}\|^{3}} e^{-\kappa\|\mathbf{y}-\mathbf{x}\|} d \sigma_{\mathbf{y}} u(\mathbf{y})\right\| \\
:= & J_{1}+J_{2}+J_{3} .
\end{aligned}
$$


Since $\partial \Omega$ is Lyapunov boundary, the normal vector $\mathbf{n}$ is continuous on $\partial \Omega$. Therefore, we can choose $0<\eta \leq 1$ such that for the scalar product

$$
(\mathbf{n}(\mathbf{x}) ; \mathbf{n}(\mathbf{y})) \geq \frac{1}{2}
$$

for all $\mathbf{x}, \mathbf{y} \in \partial \Omega$ with $\|\mathbf{y}-\mathbf{x}\| \leq \eta$. It is enough to consider the case of $\|\mathbf{y}-\mathbf{x}\|$ being sufficiently small such that the set $\partial L \triangleq\{\mathbf{y} \in \partial \Omega:\|\mathbf{y}-\mathbf{x}\| \leq \eta\}$ is connected for each $\mathbf{x} \in \partial \Omega$. Then the condition (3.35) implies that $\partial L$ can be bijective into the tangent plane to $\partial \Omega$ at the point $\mathbf{x}$. Using polar coordinates $(r, \omega)$ in the tangent plane with origin in $\mathbf{x}$, for any $u \in H^{\alpha}\left(\partial \Omega, C l\left(V_{3,3}\right)\right)$, we arrive at

$$
\begin{aligned}
\left\|\int_{\partial L} \frac{\kappa(\mathbf{y}-\mathbf{x})}{\|\mathbf{y}-\mathbf{x}\|^{2}} e^{-\kappa\|\mathbf{y}-\mathbf{x}\|} d \sigma_{\mathbf{y}} u(\mathbf{y})\right\| & \leq \kappa C_{1}\|u\|_{\infty} \int_{\partial L} \frac{1}{\|\mathbf{y}-\mathbf{x}\|} d S \leq 2 \pi \kappa C_{2}\|u\|_{\infty} \int_{0}^{\eta} d r \\
& =2 \pi \kappa C_{2} \eta\|u\|_{\infty}
\end{aligned}
$$

where $C_{1}, C_{2}$ denote nonnegative constants which are independent of $u$. Here we use the facts that $\|\mathbf{x}-\mathbf{y}\| \geq r$, that the surface element

$$
d S=\frac{r d r d \omega}{(\mathbf{n}(\mathbf{x}), \mathbf{x}(\mathbf{y}))}
$$

can be estimated with the aid of (3.35) by $d S \leq 2 r d r d \omega$, and that the projection $\partial L$ into the tangent plane is contained in the interior of the sphere of radius $\eta$ and center $\mathbf{x}$. Furthermore,

$$
\begin{aligned}
\left\|\int_{\partial \Omega \backslash \partial L} \frac{\kappa(\mathbf{y}-\mathbf{x})}{\|\mathbf{y}-\mathbf{x}\|^{2}} e^{-\kappa\|\mathbf{y}-\mathbf{x}\|} d \sigma_{\mathbf{y}} u(\mathbf{y})\right\| & \leq 2 \pi \kappa C_{3}\|u\|_{\infty} \int_{\partial \Omega \backslash \partial L} \eta^{-1} d S \\
& \leq 2 \pi \kappa C_{3}\|u\|_{\infty} \eta^{-1}|\partial \Omega|,
\end{aligned}
$$

where $|\partial \Omega|$ is the surface area of $\Omega$. Inequalities (3.36) with (3.38) imply

$$
J_{1} \leq \frac{\kappa C_{2} \eta^{2}+\kappa C_{3}|\partial \Omega|}{\eta}\|u\|_{\infty} .
$$

Using a similar method to the proof of $J_{1}$, we obtain

$$
J_{2} \leq \frac{\kappa C_{2} \eta^{2}+\kappa C_{3}|\partial \Omega|}{\eta}\|u\|_{\infty} .
$$

Now we estimate $J_{3}$. Combining $u \in H^{\alpha}\left(\partial \Omega, C l\left(V_{3,3}\right)\right)$ with

$$
\frac{1}{4 \pi} \int_{\partial \Omega} \frac{\mathbf{y}-\mathbf{x}}{\|\mathbf{y}-\mathbf{x}\|^{3}} d \sigma_{\mathbf{y}}=\frac{1}{2}, \quad \text { for } \mathbf{x} \in \partial \Omega,
$$

we have

$$
\begin{aligned}
J_{3} \leq & \left\|\frac{1}{2 \pi} \int_{\partial \Omega} \frac{\mathbf{y}-\mathbf{x}}{\|\mathbf{y}-\mathbf{x}\|^{3}} d \sigma_{\mathbf{y}}\left[e^{-\kappa\|\mathbf{y}-\mathbf{x}\|}(u(\mathbf{y})-u(\mathbf{x}))\right]\right\| \\
& +\left\|\frac{1}{2 \pi} \int_{\partial \Omega} \frac{\mathbf{y}-\mathbf{x}}{\|\mathbf{y}-\mathbf{x}\|^{3}} d \sigma_{\mathbf{y}}\left[u(\mathbf{x}) e^{-\kappa\|\mathbf{y}-\mathbf{x}\|}-u(\mathbf{x})\right]\right\|+\|u(\mathbf{x})\|
\end{aligned}
$$




$$
\begin{aligned}
\leq & C_{4}\|u\|_{\alpha} \frac{1}{2 \pi} \int_{\partial \Omega} \frac{1}{\|\mathbf{y}-\mathbf{x}\|^{2-\alpha}} d S+C_{5} \frac{1}{2 \pi}\|u\|_{\infty} \int_{\partial \Omega} \frac{1}{\|\mathbf{y}-\mathbf{x}\|} d S+\|u\|_{\infty} \\
\leq & \frac{C_{4}}{2 \pi}\left(\int_{\partial \Omega \backslash \partial L} \frac{1}{\|\mathbf{y}-\mathbf{x}\|^{2-\alpha}} d S+\int_{\partial L} \frac{1}{\|\mathbf{y}-\mathbf{x}\|^{2-\alpha}} d S\right)\|u\|_{\alpha} \\
& +\frac{C_{5}}{2 \pi}\left(\int_{\partial \Omega \backslash \partial L} \frac{1}{\|\mathbf{y}-\mathbf{x}\|} d S+\int_{\partial L} \frac{1}{\|\mathbf{y}-\mathbf{x}\|} d S\right)\|u\|_{\infty}+\|u\|_{\infty} \\
\leq & \frac{C_{4}}{2 \pi}\left(\eta^{2-\alpha}|\partial \Omega|+\frac{\eta^{2-\alpha}}{2-\alpha}\right)\|u\|_{\alpha}+\frac{C_{5}}{2 \pi}\left(\eta^{-1}|\partial \Omega|+\eta+1\right)\|u\|_{\infty} .
\end{aligned}
$$

Combining (3.34), (3.39), (3.40), and (3.41), we get

$$
\begin{aligned}
\left\|\mathbb{S}_{\kappa}[u]\right\|_{\infty} \leq & \frac{C_{4}}{2 \pi} \eta^{2-\alpha}\left(|\partial \Omega|+\frac{1}{2-\alpha}\right)\|u\|_{\alpha} \\
& +\left[\frac{C_{5}\left(|\partial \Omega|+\eta^{2}+\eta\right)}{2 \pi \eta}+\frac{2 \kappa\left(C_{2} \eta^{2}+C_{3}|\partial \Omega|\right)}{\eta}\right]\|u\|_{\infty},
\end{aligned}
$$

where $C_{4}, C_{5}, C_{6}$ denote nonnegative constants which are independent of $u$.

On the other hand, for $\mathbf{x}_{1}, \mathbf{x}_{2} \in \partial \Omega$, it is enough to consider the case of $\left\|\mathbf{x}_{1}-\mathbf{x}_{2}\right\|$ being sufficiently small. It is obvious that

$$
e^{-\kappa\left\|\mathbf{y}-\mathbf{x}_{i}\right\|} u(\mathbf{y}) \in H^{\alpha}\left(\partial \Omega \times \partial \Omega, C l\left(V_{3,3}\right)\right), \quad i=1,2,
$$

and

$$
\|\mathbf{y}-\mathbf{x}\| e^{-\kappa\left\|\mathbf{y}-\mathbf{x}_{i}\right\|} u(\mathbf{y}) \in H^{\alpha}\left(\partial \Omega \times \partial \Omega, C l\left(V_{3,3}\right)\right), \quad i=1,2 .
$$

Applying some properties of the Hilbert transform in Clifford analysis (see $[8,9,16,30]$ ) and the weak singularity of $\frac{\mathbf{y}-\mathbf{x}}{\|\mathbf{y}-\mathbf{x}\|^{2}}$ and $\frac{1}{\|\mathbf{y}-\mathbf{x}\|}$, we conclude

$$
\left\|\mathbb{S}_{\kappa}[u]\left(\mathbf{x}_{1}\right)-\mathbb{S}_{\kappa}[u]\left(\mathbf{x}_{2}\right)\right\| \leq C_{6}\|u\|_{\alpha}\left\|\mathbf{x}_{1}-\mathbf{x}_{2}\right\|^{\alpha},
$$

where $C_{6}$ is a nonnegative constant independent of $\mathbf{x}_{1}$ and $\mathbf{x}_{2}$. It follows from (3.42) and (3.43) that

$$
\left\|\mathbb{S}_{\kappa}[u]\right\|_{(\alpha, \partial \Omega)} \leq C\|u\|_{(\alpha, \partial \Omega)}
$$

where $C=\max \left\{\frac{C_{4}}{2 \pi} \eta^{2-\alpha}\left(|\partial \Omega|+\frac{1}{2-\alpha}\right)+C_{6}, \frac{C_{5}\left(|\partial \Omega|+\eta^{2}+\eta\right)}{2 \pi \eta}+\frac{2 \kappa\left(C_{2} \eta^{2}+C_{3}|\partial \Omega|\right)}{\eta}\right\}$. The proof is complete.

Remark 3.13 By the same technique we obtain the following result that the generalized Cauchy integral operator $\mathbb{S}_{-\kappa}: H^{\alpha}\left(\partial \Omega, C l\left(V_{3,3}\right)\right) \mapsto H^{\alpha}\left(\partial \Omega, C l\left(V_{3,3}\right)\right)$ defined by (3.22) is bounded.

Remark 3.14 We assume $u \in H^{\alpha}\left(\partial \Omega, C l\left(V_{3,3}\right)\right)$. All integrals are understood in the Riemann integral sense in Lemma 3.9 and Theorem 3.12. Now, let $L^{p}\left(\partial \Omega, C l\left(V_{3,3}\right)\right), 1 \leq p<\infty$ be the space of all Clifford algebra valued functions, whose $p$ th power is Lebesgue integrable in $\partial \Omega$. If $u \in L^{p}\left(\partial \Omega, C l\left(V_{3,3}\right)\right)$ then one has to understand $\mathbb{F}_{ \pm \kappa}$ as a Lebesgue integral, 
and the necessary changes can be easily made. For instance, the limits exist almost everywhere on $\partial \Omega$ with respect to the surface Lebesgue measure in Lemma 3.9. Using classical Calderón-Zygmund theory, an $L^{p}$ formulation of Theorem 3.12 holds.

In the framework of Clifford algebra $\mathrm{Cl}\left(V_{3,3}\right)$, we come back to the modified Helmholtz equation $\left(\Delta-\kappa^{2}\right)[u](\mathbf{x})=0, \mathbf{x} \in \Omega$. By Theorem 3.3, Theorem 3.4, Theorem 3.10, and Theorem 3.11, we have the following theorem.

Theorem 3.15 Suppose that $\Omega$ is an open nonempty bounded subset of $\mathbb{R}^{3}$ with a Lyapunov boundary $\partial \Omega, f, g \in C^{1}\left(\Omega, C l\left(V_{3,3}\right)\right) \cap C\left(\bar{\Omega}, C l\left(V_{3,3}\right)\right), L_{-\kappa}[f]=0$ and $L_{\kappa}[g]=0$ in $\Omega$. Then the function $u(\mathbf{x})$ is determined by

$$
u(\mathbf{x})=\mathbb{T}_{\kappa}[f](\mathbf{x})+g(\mathbf{x})
$$

or

$$
u(\mathbf{x})=\mathbb{T}_{-\kappa}[g](\mathbf{x})+f(\mathbf{x}) .
$$

Conversely, suppose $u(\mathbf{x}) \in C^{1}\left(\bar{\Omega}, C l\left(V_{3,3}\right)\right)$ and $u(\mathbf{x})$ is a solution of the modified Helmholtz equation. Then $u$ may be represented by (3.44) or (3.45), where $L_{-\kappa}[f]=0$ and $L_{\kappa}[g]=0$ in $\Omega$.

\section{Some boundary value problems for modified Helmholtz equations and its application}

We consider the following Riemann type problem now:

$$
\begin{cases}H u=0, & \text { in } \mathbb{R}^{3} \backslash \partial \Omega, \\ u^{+}(\mathbf{x})=u^{-}(\mathbf{x}) f(\mathbf{x})+g_{1}(\mathbf{x}), & \mathbf{x} \in \partial \Omega, \\ L_{\kappa}[u]^{+}(\mathbf{x})=L_{\kappa}[u]^{-}(\mathbf{x}) A+g_{2}(\mathbf{x}), & \mathbf{x} \in \partial \Omega, \\ \lim _{\|\mathbf{x}\| \rightarrow \infty} u(\mathbf{x})=0, & \end{cases}
$$

where $A$ is any invertible Clifford constant. $g_{1}(\mathbf{x}), g_{2}(\mathbf{x})$, and $f(\mathbf{x})$ are Clifford value functions in $H^{\alpha}\left(\partial \Omega, C l\left(V_{3,3}\right)\right), 0<\alpha<1, H=\Delta-\kappa^{2}, \kappa \geq 0$. We establish solvability conditions of the Riemann type problem (4.1).

Theorem 4.1 Suppose $f(\mathbf{x}), g_{1}(\mathbf{x}), g_{2}(\mathbf{x}) \in H^{\alpha}\left(\partial \Omega, C l\left(V_{3,3}\right)\right), 0<\alpha<1$, and $f(\mathbf{x})$ satisfies the following condition:

$$
\|1-f(\mathbf{x})\|_{(\alpha, \partial \Omega)}<\frac{1}{C+1}
$$

where $C$ is a positive constant mentioned in Theorem 3.12. Then there exists a unique solution to the Riemann type problem (4.1).

Proof Combining $H[u](\mathbf{x})=L_{-\kappa}\left[L_{\kappa}[u]\right](\mathbf{x})=0$ and $u(\infty)=0$, we easily check $L_{-\kappa}[u](\mathbf{x})=0$ and $\omega(\infty)=0$. Let $\omega(\mathbf{x})=L_{\kappa}[u](\mathbf{x})$. Then

$$
\omega^{+}(\mathbf{x})=\omega^{-}(\mathbf{x}) A+g_{2}(\mathbf{x}), \quad \mathbf{x} \in \partial \Omega
$$


holds. Furthermore, we obtain

$$
\omega(\mathbf{x})= \begin{cases}\int_{\partial \Omega} K_{1}(\mathbf{x}, \mathbf{y}, \kappa) d \sigma_{y} g_{2}(\mathbf{y}), & \mathbf{x} \in \Omega^{+} \\ \int_{\partial \Omega} K_{1}(\mathbf{x}, \mathbf{y}, \kappa) d \sigma_{y} g_{2}(\mathbf{y}) A^{-1}, & \mathbf{x} \in \Omega^{-}\end{cases}
$$

Let

$$
u_{1}(x)= \begin{cases}\frac{1}{4 \pi} \int_{\partial \Omega} \frac{e^{-\kappa\|\mathbf{y}-\mathbf{x}\|}}{\|\mathbf{y} \mathbf{x}\|} d \sigma_{y} g_{2}(\mathbf{y}), & \mathbf{x} \in \Omega^{+}, \\ \frac{1}{4 \pi} \int_{\partial \Omega} \frac{e^{-\kappa \mid \mathbf{y}-\mathbf{x} \|}}{\|\mathbf{y}-\mathbf{x}\|} d \sigma_{y} g_{2}(\mathbf{y}) A^{-1}, & \mathbf{x} \in \Omega^{-} .\end{cases}
$$

It is easy to see that

$$
L_{\kappa}\left[u-u_{1}\right](\mathbf{x})=0, \quad \mathbf{x} \in \mathbb{R}^{3} \backslash \partial \Omega .
$$

Denote $u(\mathbf{x})-u_{1}(\mathbf{x}):=\varphi(\mathbf{x}), \mathbf{x} \in \mathbb{R}^{3} \backslash \partial \Omega$, applying the transmission condition

$$
u^{+}(\mathbf{x})=u^{-}(\mathbf{x}) f(\mathbf{x})+g_{1}(\mathbf{x}), \quad \mathbf{x} \in \partial \Omega,
$$

we have

$$
\varphi^{+}(\mathbf{x})=\varphi^{-}(\mathbf{x}) f(\mathbf{x})+\widetilde{g}_{1}(\mathbf{x}), \quad \mathbf{x} \in \partial \Omega,
$$

where

$$
\widetilde{g}_{1}(\mathbf{x})=g_{1}(\mathbf{x})-\frac{1}{\omega_{3}} \int_{\partial \Omega} \frac{e^{-\kappa\|\mathbf{y}-\mathbf{x}\|}}{\|\mathbf{y}-\mathbf{x}\|} d \sigma_{y} g_{2}(\mathbf{y})+\frac{1}{\omega_{3}} \int_{\partial \Omega} \frac{e^{-\kappa\|\mathbf{y}-\mathbf{x}\|}}{\|\mathbf{y}-\mathbf{x}\|} d \sigma_{y} g_{2}(\mathbf{y}) A^{-1} f(\mathbf{x}) .
$$

It is clear that $\widetilde{g}_{1}(\mathbf{x}) \in H^{\alpha}\left(\partial \Omega, C l\left(V_{3,3}\right)\right), 0<\alpha<1$. We have $\varphi(\infty)=0$. Combining (4.6) with (4.7), we have

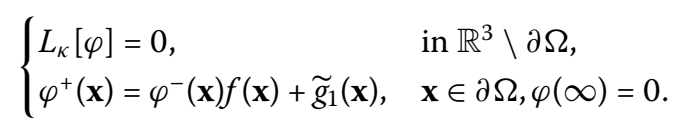

We only need to consider the existence of solutions to (4.8). The solution to this problem may be written in the form

$$
\varphi(\mathbf{x})=\int_{\partial \Omega} K_{* 1}(\mathbf{x}, \mathbf{y}, \kappa) d \sigma_{y} \varphi_{1}(\mathbf{y}),
$$

where $\varphi_{1}(\mathbf{y})$ is a Hölder continuous function to be determined on $\partial \Omega$. Using Lemma 3.9, (4.8) can be reduced to an equivalent singular integral equation for $\varphi_{1}$,

$$
\varphi_{1}(\mathbf{x})=\left[\frac{\varphi_{1}(\mathbf{x})}{2}-\int_{\partial \Omega} K_{* 1}(\mathbf{x}, \mathbf{y}, \kappa) d \sigma_{y} \varphi_{1}(\mathbf{y})\right](1-f(\mathbf{x}))+\widetilde{g}_{1}(\mathbf{x}) .
$$

We set

$$
\left(T \varphi_{1}\right)(\mathbf{x})=\left[\varphi_{1}(\mathbf{x})-\left(\mathbb{S}_{\kappa} \varphi_{1}\right)(\mathbf{x})\right] \frac{(1-f(\mathbf{x}))}{2}+\widetilde{g}_{1}(\mathbf{x}) .
$$


For any $\omega_{1}, \omega_{2} \in H^{\alpha}\left(\partial \Omega, C l\left(V_{3,3}\right)\right)$,

$$
\left\|T \omega_{1}-T \omega_{2}\right\|_{(\alpha, \partial \Omega)} \leq\left\|\omega_{1}-\omega_{2}\right\|_{(\alpha, \partial \Omega)}\|1-f\|_{(\alpha, \partial \Omega)}(1+C) .
$$

From (4.2), the integral operator $T$ is a contraction operator mapping the Banach space $H^{\alpha}\left(\partial \Omega, C l\left(V_{3,3}\right)\right)$ into itself. Then the operator $T$ has a unique fixed point. Thus there exists a unique solution to (4.8). The proof is finished.

Remark 4.2 In the above Theorem 4.1, the existence and uniqueness of solutions of the Riemann type problem for the modified Helmholtz equation with variable coefficient i.e., $f(\mathbf{x}) \in H^{\alpha}\left(\partial \Omega, C l\left(V_{3,3}\right)\right)$ is illustrated. Particularly, when $f(\mathbf{x})$ is just an invertible Clifford constant, for the boundary value problem (4.1) there exists a unique solution. Moreover, we have obtained an explicit representation of solutions in [22].

As applications, using Lemma 3.9 and the results of the boundary value problem, we consider two kinds of singular integral equations and obtain their explicit representations of solutions.

Theorem 4.3 Consider the singular integral equation:

$$
u(\mathbf{x}) A+2 \int_{\partial \Omega} K_{* 1}(\mathbf{x}, \mathbf{y}, \kappa) d \sigma_{\mathbf{y}} u(\mathbf{y}) B=f(\mathbf{x}), \quad \mathbf{x} \in \partial \Omega \subset \mathbb{R}^{3},
$$

where $f(\mathbf{x}) \in H^{\alpha}\left(\partial \Omega, C l\left(V_{3,3}\right)\right), B$ is a non-zero Clifford constant, $A+B$ and $A-B$ are invertible Clifford constants, and $(A+B)^{-1}$ and $(A-B)^{-1}$ are invertible elements, respectively. Then:

1. If $u(\mathbf{x}) \in H^{\alpha}\left(\partial \Omega, C l\left(V_{3,3}\right)\right)$ is a solution to (4.13), and

$$
\mathfrak{F}_{* 1}(\mathbf{x})=\int_{\partial \Omega} K_{* 1}(\mathbf{x}, \mathbf{y}, \kappa) d \sigma_{\mathbf{y}} u(\mathbf{y}), \quad \mathbf{x} \in \mathbb{R}^{3} \backslash \partial \Omega,
$$

then

$$
\mathfrak{F}_{* 1}^{+}(\mathbf{x})=\mathfrak{F}_{* 1}^{-}(\mathbf{x}) \cdot(A-B)(A+B)^{-1}+f(\mathbf{x})(A+B)^{-1}, \quad \mathbf{x} \in \partial \Omega
$$

and

$$
\lim _{\|\mathbf{x}\| \rightarrow \infty} \mathfrak{F}_{* 1}(\mathbf{x})=0
$$

2. Assume $\mathfrak{F}_{* 1}(\mathbf{x})$ is solution of Riemann type problem (4.15) and $L_{\kappa}\left[\mathfrak{F}_{* 1}\right](\mathbf{x})=0$, $\mathbf{x} \in \mathbb{R}^{3} \backslash \partial \Omega, \mathfrak{F}_{* 1}^{+}(\mathbf{x}), \mathfrak{F}_{* 1}^{-}(\mathbf{x}) \in H^{\alpha}\left(\partial \Omega, C l\left(V_{3,3}\right)\right)$. Let

$$
u(\mathbf{x})=\mathfrak{F}_{* 1}^{+}(\mathbf{x})-\mathfrak{F}_{* 1}^{-}(\mathbf{x})
$$

Then $u(\mathbf{x})$ is the solution of the singular equation (4.13) $u(\mathbf{x}) \in H^{\alpha}\left(\partial \Omega, C l\left(V_{3,3}\right)\right)$.

Proof 1. Using Lemma 3.9, we have

$$
\mathfrak{F}_{* 1}^{+}(\mathbf{x})=\frac{u(\mathbf{x})}{2}+\int_{\partial \Omega} K_{* 1}(\mathbf{x}, \mathbf{y}, \kappa) d \sigma_{\mathbf{y}} u(\mathbf{y})
$$




$$
\mathfrak{F}_{* 1}^{+}(\mathbf{x})=-\frac{u(\mathbf{x})}{2}+\int_{\partial \Omega} K_{* 1}(\mathbf{x}, \mathbf{y}, \kappa) d \sigma_{\mathbf{y}} u(\mathbf{y})
$$

Combining (4.17) with (4.18), we get

$$
\left[\mathfrak{F}_{* 1}^{+}(\mathbf{x})-\mathfrak{F}_{* 1}^{-}(\mathbf{x})\right] A+\left[\mathfrak{F}_{* 1}^{+}(\mathbf{x})+\mathfrak{F}_{* 1}^{-}(\mathbf{x})\right] B=f(\mathbf{x})
$$

i.e.,

$$
\mathfrak{F}_{* 1}^{+}(\mathbf{x})=\mathfrak{F}_{* 1}^{-}(\mathbf{x}) \cdot(A-B)(A+B)^{-1}+f(\mathbf{x})(A+B)^{-1}, \quad \mathbf{x} \in \partial \Omega .
$$

It is clear that $\mathfrak{F}(\infty)=0$. The result follows.

2. In view of Theorem 4.1 and Remark 4.2, we obtain

$$
\mathfrak{F}_{* 1}(\mathbf{x})=\int_{\partial \Omega} K_{* 1}(\mathbf{x}, \mathbf{x}, \kappa) d \sigma_{\mathbf{y}} u(\mathbf{y})
$$

By Lemma 3.9, we get

$$
\begin{aligned}
& \mathfrak{F}_{* 1}^{+}(\mathbf{x})=\frac{u(\mathbf{x})}{2}+\int_{\partial \Omega} K_{* 1}(\mathbf{x}, \mathbf{y}, \kappa) d \sigma_{\mathbf{y}} u(\mathbf{y}), \\
& \mathfrak{F}_{* 1}^{-}(\mathbf{x})=-\frac{u(\mathbf{x})}{2}+\int_{\partial \Omega} K_{* 1}(\mathbf{x}, \mathbf{y}, \kappa) d \sigma_{\mathbf{y}} u(\mathbf{y}) .
\end{aligned}
$$

Combining (4.22), (4.23), and (4.20), the result follows.

Theorem 4.4 The singular integral equation (4.13) is solvable in $H^{\alpha}\left(\partial \Omega, C l\left(V_{3,3}\right)\right)$ and the solution may be represented by the following formula:

$$
\begin{aligned}
u(\mathbf{x})= & \frac{f(\mathbf{x})}{2}\left[(A+B)^{-1}+(A-B)^{-1}\right] \\
& +\int_{\partial \Omega} K_{* 1}(\mathbf{x}, \mathbf{y}, \kappa) d \sigma_{\mathbf{y}} f(\mathbf{y})\left[(A+B)^{-1}-(A-B)^{-1}\right], \quad \mathbf{x} \in \partial \Omega .
\end{aligned}
$$

Proof In view of Theorem 4.3, we consider the following Riemann boundary value problem:

$$
\begin{cases}L_{\kappa}[\mathfrak{F}]=0, & \text { in } \mathbb{R}^{3} \backslash \partial \Omega \\ \mathfrak{F}_{* 1}^{+}(\mathbf{x})=\mathfrak{F}_{* 1}^{-}(\mathbf{x}) \cdot(A-B)(A+B)^{-1}+f(\mathbf{x})(A+B)^{-1}, & \mathbf{x} \in \partial \Omega \\ \mathfrak{F}_{* 1}(\infty)=0 & \end{cases}
$$

We have

$$
\mathfrak{F}_{* 1}(\mathbf{x})= \begin{cases}\int_{\partial \Omega} K_{* 1}(\mathbf{x}, \mathbf{y}, \kappa) d \sigma_{\mathbf{y}} f(\mathbf{y})(A+B)^{-1}, & \mathbf{x} \in \Omega^{+} \\ \int_{\partial \Omega} K_{* 1}(\mathbf{x}, \mathbf{y}, \kappa) d \sigma_{\mathbf{y}} f(\mathbf{y})(A-B)^{-1}, & \mathbf{x} \in \Omega^{-}\end{cases}
$$

Using again Theorem 4.3, the proof is complete. 
Remark 4.5 When $A=0$, the singular integral equation (4.13) is solvable in $H^{\alpha}(\partial \Omega$, $\left.C l\left(V_{3,3}\right)\right)$ and the solution may be represented by the following formula:

$$
u(\mathbf{x})=2 \int_{\partial \Omega} K_{* 1}(\mathbf{x}, \mathbf{y}, \kappa) d \sigma_{\mathbf{y}} f(\mathbf{y}) B^{-1}, \quad \mathbf{x} \in \partial \Omega .
$$

The following theorems can be similarly proved as Theorem 4.3 and Theorem 4.4.

Theorem 4.6 Consider the singular integral equation:

$$
u(\mathbf{x}) C+2 \int_{\partial \Omega} K_{1}(\mathbf{x}, \mathbf{y}, \kappa) d \sigma_{\mathbf{y}} u(\mathbf{y}) D=f(\mathbf{x}), \quad \mathbf{x} \in \partial \Omega \subset \mathbb{R}^{3},
$$

where $f(\mathbf{x}) \in H^{\alpha}\left(\partial \Omega, C l\left(V_{3,3}\right)\right), D$ is a non-zero Clifford constant, $C+D$ and $C-D$ are invertible Clifford constants, and $(C+D)^{-1}$ and $(C-D)^{-1}$ are invertible elements, respectively. Then:

1. If $u(\mathbf{x}) \in H^{\alpha}\left(\partial \Omega, C l\left(V_{3,3}\right)\right)$ is a solution to (4.27), set

$$
\mathfrak{F}_{1}(\mathbf{x})=\int_{\partial \Omega} K_{1}(\mathbf{x}, \mathbf{y}, \kappa) d \sigma_{\mathbf{y}} u(\mathbf{y}), \quad \mathbf{x} \in \mathbb{R}^{3} \backslash \partial \Omega .
$$

Then

$$
\mathfrak{F}_{1}^{+}(\mathbf{x})=\mathfrak{F}_{1}^{-}(\mathbf{x}) \cdot(A-B)(A+B)^{-1}+f(\mathbf{x})(A+B)^{-1}, \quad \mathbf{x} \in \partial \Omega
$$

and

$$
\lim _{\|\mathbf{x}\| \rightarrow \infty} \mathfrak{F}_{1}(\mathbf{x})=0
$$

2. Conversely, if $\mathfrak{F}_{1}(\mathbf{x})$ is solution of Riemann type problem (4.29) and $L_{-\kappa}[\mathfrak{F}](\mathbf{x})=0$, $\mathbf{x} \in \mathbb{R}^{3} \backslash \partial \Omega, \mathfrak{F}_{1}^{+}(\mathbf{x}), \mathfrak{F}_{1}^{-}(\mathbf{x}) \in H^{\alpha}\left(\partial \Omega, C l\left(V_{3,3}\right)\right)$. Let

$$
u(\mathbf{x})=\mathfrak{F}_{1}^{+}(\mathbf{x})-\mathfrak{F}_{1}^{-}(\mathbf{x})
$$

Then $u(\mathbf{x})$ is the solution of singular equation (4.27), $u(\mathbf{x}) \in H^{\alpha}\left(\partial \Omega, C l\left(V_{3,3}\right)\right)$.

Theorem 4.7 The singular integral equation (4.27) is solvable in $H^{\alpha}\left(\partial \Omega, C l\left(V_{3,3}\right)\right)$ and the solution may be represented by the following formula:

$$
\begin{aligned}
u(\mathbf{x})= & \frac{f(\mathbf{x})}{2}\left[(C+D)^{-1}+(C-D)^{-1}\right] \\
& +\int_{\partial \Omega} K_{1}(\mathbf{x}, \mathbf{y}, \kappa) d \sigma_{\mathbf{y}} f(\mathbf{y})\left[(C+D)^{-1}-(C-D)^{-1}\right], \quad \mathbf{x} \in \partial \Omega .
\end{aligned}
$$

Remark 4.8 When $C=0$, the singular integral equation (4.27) is solvable in $H^{\alpha}(\partial \Omega$, $\left.C l\left(V_{3,3}\right)\right)$ and the solution may be represented by the following formula:

$$
u(\mathbf{x})=2 \int_{\partial \Omega} K_{*}(\mathbf{x}, \mathbf{y}, \kappa) d \sigma_{\mathbf{y}} f(\mathbf{y}) D^{-1}, \quad \mathbf{x} \in \partial \Omega
$$




\section{Competing interests}

The authors declare that they have no competing interests.

\section{Authors' contributions}

All authors contributed equally to the manuscript and typed, read, and approved the final manuscript.

\section{Acknowledgements}

The authors cordially thank the referees for their careful reading and helpful comments. This work was partially supported by NNSF of China (Nos. 11401287 and 11271175), the AMEP and DYSP of Linyi University.

\section{Received: 6 September 2015 Accepted: 12 November 2015 Published online: 25 November 2015}

\section{References}

1. Abreu Blaya, R, Bory Reyes, J: On the Riemann Hilbert type problems in Clifford analysis. Adv. Appl. Clifford Algebras $11,15-26(2001)$

2. Abreu Blaya, R, Bory Reyes, J, Peña-Peña, D: Jump problem and removable singularities for monogenic functions. J. Geom. Anal. 17, 1-13 (2007)

3. Abreu Blaya, R, Bory Reyes, J, Brackx, F, De Schepper, H, Sommen, F: Boundary value problems associated to a Hermitian Helmholtz equation. J. Math. Anal. Appl. 389, 1268-1279 (2012)

4. Antipov, Y, Fokas, AS: A transform method for the modified Helmholtz equation on the semi-strip. Math. Proc. Camb. Philos. Soc. 137, 339-365 (2004)

5. ben-Avraham, D, Fokas, AS: The solution of the modified Helmholtz equation in a wedge and an application to diffusion-limited coalescence. Phys. Lett. A 263, 355-395 (1999)

6. ben-Avraham, D, Fokas, AS: Solution of the modified Helmholtz equation in a triangular and an application to diffusion-limited coalescence. Phys. Rev. E 64(3), 016114 (2001)

7. Bernstein, S: On the left linear Riemann problem in Clifford analysis. Bull. Belg. Math. Soc. Simon Stevin 3, 557-576 (1996)

8. Gürlebeck, K, Sprössig, W: Quaternionic Analysis and Elliptic Boundary Value Problems. Birkhäuser, Basel (1990)

9. Gürlebeck, K, Sprössig, W: Quaternionic and Clifford Calculus for Physicists and Engineers. Wiley, Chichester (1997)

10. Jleli, M, Samet, B, Vial, G: Topological sensitivity analysis for the modified Helmholtz equation under an impedance condition on the boundary of a hole. J. Math. Pures Appl. 103, 557-574 (2015)

11. Li, X: On solving boundary value problems of modified Helmholtz equations by plane wave functions. J. Comput. Appl. Math. 195, 66-82 (2006)

12. McIntosh, A, Mitrea, M: Clifford algebras and Maxwell's equations in Lipschitz domains. Math. Methods Appl. Sci. 22, 1599-1620 (1999)

13. Mitrea, M: Boundary value problems and Hardy spaces associated to the Helmholtz equation in Lipschitz domains. J. Math. Anal. Appl. 202, 819-842 (1996)

14. Mitrea, M: Boundary value problems for Dirac operators and Maxwell's equations in nonsmooth domains. Math Methods Appl. Sci. 25, 1355-1369 (2002)

15. Kravchenko, W, Shapiro, M: Integral Representations for Spatial Models of Mathematical Physics. Res. Notes Math. vol. 351. Pitman, London (1996)

16. $\mathrm{Xu}, \mathrm{Z}$ : On linear and nonlinear Riemann-Hilbert problems for regular functions with values in Clifford algebras. Chin. Ann. Math., Ser. B 11, 349-358 (1990)

17. $\mathrm{Xu}, \mathrm{Z}$ : Helmholtz equations and boundary value problems. In: Partial Differential Equations with Complex Analysis. Pitman Res. Notes Math. Ser., vol. 262, pp. 204-214. Longman, Harlow (1992)

18. $\mathrm{Bu}, \mathrm{Y}, \mathrm{Du}, \mathrm{J}$ : The RH boundary value problem for the k-monogenic functions. J. Math. Anal. Appl. 347, 633-644 (2008)

19. Gong, Y, Du, J: A kind of Riemann and Hilbert boundary value problem for left monogenic functions in $\mathbb{R}^{m}(m \geq 2)$. Complex Var. Theory Appl. 49, 303-318 (2004)

20. Gürlebeck, K, Zhang, Z: Some Riemann boundary value problems in Clifford analysis. Math. Methods Appl. Sci. 33 287-302 (2010)

21. Gürlebeck, K, Zhang, Z: Generalized integral representations for functions with values in $C\left(V_{3,3}\right)$. Chin. Ann. Math., Ser. B 32, 123-138 (2011)

22. Gu, L, Du, J, Cai, D: A kind of Riemann boundary value problems for pseudo-harmonic functions in Clifford analysis. Complex Var. Elliptic Equ. 59, 412-426 (2014)

23. Gu, L, Zhang, Z: Riemann boundary value problem for harmonic functions in Clifford analysis. Math. Nachr. 287, 1001-1012 (2014)

24. Muskhelishvilli, NI: Singular Integral Equations. Nauka, Moscow (1968)

25. Lu, J: Boundary Value Problems of Analytic Functions. World Scientific, Singapore (1993)

26. Brackx, F, Delanghe, R, Sommen, F: Clifford Analysis. Research Notes in Mathematics, vol. 76. Pitman, London (1982)

27. Delanghe, R, Sommen, F, Souček, V: Clifford Algebras and Spinor-Valued Functions. Kluwer Academic, Dordrecht (1992)

28. Delanghe, R: On the regular analytic functions with values in a Clifford algebra. Math. Ann. 185, 91-111 (1970)

29. Delanghe, R: On the singularities of functions with values in a Clifford algebra. Math. Ann. 196, 293-319 (1972)

30. Zhang, Z: Some properties of operators in Clifford analysis. Complex Var. Elliptic Equ. 52, 455-473 (2007) 